\title{
Análisis de resultados del aprendizaje presencial y aprendizaje semipresencial en dos cursos universitarios
}

\author{
ANA DASSO VASSALLO* \\ Universidad Tecnológica del Perú - Perú \\ INÉS EVARISTO CHIYONG* \\ Universidad Tecnológica del Perú - Perú \\ Recibido el 20-11-19; primera evaluación el 08-06-20; \\ segunda evaluación el 25-06-20; aceptado el 15-07-20
}

\section{Resumen}

Este artículo describe los resultados, notas y percepciones del estudiante, obtenidos por los alumnos de dos cursos en sus modalidades presenciales y semipresenciales ofrecidos en una universidad particular de Lima. Los cursos analizados fueron Estadística Descriptiva y Probabilidades, que usa la metodología STEM, y el curso Individuo y Medio Ambiente, que no la usa. Los resultados no muestran diferencias estadísticamente significativas entre las notas de los alumnos según la modalidad en ninguno de los dos cursos y semestres en los que se trabajó, resultado que está de acuerdo con lo encontrado en la literatura. Este artículo busca ser un aporte a las discusiones y a las decisiones en torno al crecimiento de la modalidad semipresencial en Perú que se ofrece como alternativa a la educación tradicional.

Palabras clave: B-Learning, educación superior, calificaciones, educación y tecnología, enseñanza semipresencial.

\footnotetext{
* Magíster en Cognición Aprendizaje y Desarrollo por la Pontificia Universidad Católica del Perú (PUCP). Licenciada en Psicología por la PUCP. Docente principal auxiliar en la Universidad Tecnológica del Perú. Correo electrónico: fiorella.dasso@pucp.edu.pe. https://orcid.org/0000-0002-6970-0107

** Licenciada en Psicología Educacional por la Pontificia Universidad Católica del Perú (PUCP). Magíster en Enseñanza y Aprendizaje Abiertos y a Distancia por la UNED-España. Egresada de la Maestría en Políticas Educativas por la PUCP. Directora de Aprendizaje Virtual de la Universidad Tecnológica del Perú. Docente del Departamento de Psicología PUCP. Correo: ievaristo@inlearning. edu.pe. https://orcid.org/0000-0003-2308-4289
} 


\section{Comparative study: Face-to-face learning and blended learning}

\section{Abstract}

This article reviews the comparison of results, grades and students' perception between two courses in their face-to-face and blended modalities. The courses on which we worked were Descriptive and Probability Statistics, which uses the STEM methodology, and Individual and Environment, which does not. The results show no statistically significant differences between the students' grades according to modality in any of the two courses and semesters that were reviewed for this study. This is consistent with what was found in the theoretical review. This article aims to help discussions and decision making around the growth of blended learning in Peru, which presents itself as an alternative to traditional lecturing.

Keywords: B-Learning, higher education, grades, education and technology.

\section{Analises dos resultados da aprendizagem na modalidade presencial y misto em dois cursos universitários}

\section{Resumo}

Este artigo revisa a comparação entre as notas obtidas pelos alunos e sua percepção de dois cursos nas modalidades presencial e semipresencial. Os cursos trabalhados foram Estatística Descritiva e Probabilidades, que usa a metodologia STEM, e Individuo e Meio Ambiente, que não a usa. Os resultados não mostram diferenças estatisticamente significantes entre as notas dos alunos de acordo com a modalidade em nenhum dos dois cursos e semestres nos quais se trabalhou. Isso concorda com o que foi pesquisado na revisão teórica, embora enfatize nas diferenças entre os cursos STEM e não-STEM.

Palavras-chave: B-Learning, educação superior, notas, educação y tecnologia, ensino semipresencial. 


\section{INTRODUCCIÓN}

Estamos viviendo un momento de crecimiento tecnológico que abre las puertas a muchas nuevas posibilidades. Las nuevas tecnologías están cada vez más presentes en nuestras vidas e, inevitablemente, han reconfigurado gran parte de las actividades humanas: cómo y qué comemos, cuándo dormimos, con qué nos entretenemos y, por supuesto, cómo aprendemos también (Azevedo y Aleven, 2013). Este avance de la tecnología ha provocado que los distintos actores del ámbito educativo replanteen la manera en la que estudiantes y profesores deben construir el conocimiento (Mortis, Del Hierro, García y Manig, 2015).

La generación de nuevas tecnologías, su aplicación a la educación y la universalización de ciertos dispositivos han permitido que la educación no presencial se vuelva cada vez más frecuente (García-Ruiz, Aguaded y Bartolomé-Pina, 2018). Para este tipo de educación existen principalmente dos modalidades; la conocida como e-learning o aprendizaje virtual, la cual es una forma de educación a distancia que se sostiene principalmente en el uso de las tecnologías de la información y comunicación (TIC) (Khan, 2015); y la modalidad semipresencial o blended learning, que es una modalidad en la que se combinan tanto clases presenciales como clases virtuales, siendo esta última la más frecuente (García-Ruiz, Aguaded y Bartolomé-Pina, 2018).

Precisamente debido al avance de la tecnología, estas modalidades de enseñanza están teniendo cada vez un rol más protagónico en el ámbito educativo, sobre todo en espacios de educación superior o en programas de formación continua para adultos (Pei-Chen, Tsai, Finger, Chen y Yeh, 2008). Según García-Ruiz, et al. (2018) la modalidad blended se posiciona como una alternativa más acorde a las demandas actuales de los estudiantes.

\section{MarCo teórico}

La presente investigación busca describir los resultados de aprendizaje de dos cursos universitarios en dos modalidades distintas. Por ello, es necesario definirlas. Las modalidades que se compararán son la presencial y la semipresencial. Cuando hablamos de modalidad presencial nos referimos a la educación tradicional donde el alumno y el docente se encuentran en un mismo lugar y en momento determinado para gran parte del proceso y la acción educativa; sin embargo, esto no limita el hecho de que la clase pueda verse enriquecida con las tecnologías, siendo estas un complemento (García-Aretio, 2020). Por otra parte, el blended-learning, también llamado modalidad semipresencial, es la convergencia entre lo presencial y lo virtual. Este término hace referencia 
a una modalidad donde existen momentos presenciales y momentos a distancia definidos por un diseño instruccional donde se delimitan las actividades que se llevarán a cabo de manera virtual o a distancia y aquellas que se realizarán con el docente (García-Aretio, 2018).

Ahora bien, la definición de aprendizaje semipresencial puede resultar muy amplia y ambigua, pues si lo entendemos como la simple combinación entre actividades presenciales y virtuales, un curso en el que todas las clases son presenciales y las evaluaciones son virtuales respondería a esta modalidad. Sin embargo, Graham (2006) y Khan (2015) plantean que el blended learning no consiste en la combinación aleatoria de lo presencial y lo virtual, sino que lo fundamental de esta modalidad es una cuidadosa toma de decisiones respecto a qué actividades es conveniente ejecutar presencialmente y cuáles necesitan apoyarse en recursos virtuales. Por ello, la fase de diseño del curso, y por ende el rol del profesor, son fundamentales para que el aprendizaje semipresencial sea una experiencia beneficiosa para los estudiantes. Así, la fase de diseño del curso, el nivel de involucramiento por parte del docente y la capacidad de trabajar autónomamente de los estudiantes son los principales desafíos que enfrenta el blended learning (Mortis et al., 2015)

Una vez definidas las modalidades de educación revisaremos las principales ventajas de la modalidad semipresencial. La primera ventaja es que esta modalidad permite que estudiantes y profesores lidien con las limitaciones de espacio y tiempo adaptándose a las necesidades del estudiante (García-Aretio, 2020). Otra gran ventaja del e-learning y el blended learning es que promueven la autonomía de los estudiantes en tanto requieren bastante trabajo individual y, por otro lado, también favorecen las interacciones constantes entre estudiantes y entre ellos y los instructores, pues ofrecen plataformas que facilitan la comunicación.

Aunque el e-learning es una alternativa de enseńanza muy atractiva, acorde a las demandas del siglo XXI con múltiples ventajas para los estudiantes, el aprendizaje cien por ciento virtual no ha podido desplazar al tradicional o presencial por la falta de interacción social cara a cara, la cual representa ganancias a nivel de habilidades comunicativas, protege del aislamiento a los estudiantes (Al-Qahtani y Higgins, 2012) y, por ende, les permite mantener el interés en el curso (Mortis et al., 2015). Ante este panorama y en un intento por recoger los beneficios de las modalidades presencial y virtual y así superar sus limitaciones, el blended learning surge como una alternativa aún más atractiva (Mortis et al., 2015).

El blended learning es considerado una modalidad híbrida, pues combina y balancea los recursos más efectivos de la modalidad presencial y la virtual (Zhang y Zhu, 2017). Así, el aprendizaje toma lugar tanto en espacios físicos 
como virtuales, más aún, el aula virtual funciona como una extensión del aula física (Zhang y Zhu, 2017). Esta modalidad tiene la finalidad de servir mejor las necesidades de los estudiantes y, sobre todo, brindarles una mayor diversidad de oportunidades de aprendizaje y así potenciar sus procesos educativos (Khan, 2015).

Por otra parte, en cuanto a los resultados de la modalidad blended, la investigación empírica ha encontrado que esta tiene buena recepción por parte de los estudiantes, pues la mayoría de ellos reportan sentirse satisfechos con la misma. Larson y Chung-Hsien (2009) realizaron una encuesta a 168 estudiantes universitarios estadounidenses inscritos en un curso introductorio ofrecido en tres modalidades por el mismo instructor: presencial, virtual y semipresencial. Los autores encontraron que más de la mitad de los estudiantes señalaron que las modalidades semipresencial y virtual aumentaron su interés por la materia, su pensamiento crítico y el nivel de esfuerzo puesto en el curso. Los autores explican que esta diferencia puede estar asociada a los foros virtuales, en los cuales los estudiantes tienen que discutir permanentemente sobre temas propuestos por el instructor, una actividad que no está presente en la modalidad presencial. Señalan que, si bien presencialmente también se promueve la discusión, es más difícil que todos los estudiantes participen.

Por su parte, Mortis et al. (2015) llevaron a cabo una investigación mixta con estudiantes mexicanos de educación inscritos en las licenciaturas de educación y educación infantil, cuya finalidad era recoger sus percepciones acerca de la modalidad semipresencial. Los autores encontraron que la decisión de los estudiantes por optar por la modalidad semipresencial depende de la materia puesto que consideran que hay materias que requieren de mayor práctica y, por ende, necesitan de mayor apoyo presencial. En esta investigación también se encontró que los alumnos que habían llevado más cursos en la modalidad semipresencial tendían a puntuarla mejor que aquellos que no habían llevado cursos semipresenciales anteriormente, especialmente en la comunicación con sus docentes y retroalimentación.

En cuanto a los resultados de aprendizaje que, probablemente es el tema de mayor interés para profesores y estudiantes, los estudios empíricos también han encontrado buena evidencia para respaldar la implementación de la modalidad semipresencial, pues los estudiantes obtienen notas similares o mejores que sus pares de la modalidad tradicional. Por ejemplo, en el estudio comparativo de Larson y Chung-Hsien (2009) descrito anteriormente, se compararon las calificaciones de los estudiantes de las tres modalidades. Tras un análisis de varianza, no se encontraron diferencias significativas ni en las notas parciales ni en las finales de los participantes. 
Vo, Zhu y Diep (2017) realizaron un metanálisis en el que incorporaron 51 investigaciones $(k=51)$ hechas alrededor del mundo entre 2001 y 2015. En este estudio, se compararon las calificaciones finales de estudiantes de educación superior que tomaron cursos en las modalidades presencial y semipresencial. Se encontró un efecto promedio de 0,327 en la condición semipresencial, que es de un tamaño moderado según los criterios de Cohen (1988, citado por Vo et al., 2017). Es decir, la nota de los estudiantes de los cursos semipresenciales es 0,327 desviaciones estándar más alta que la de sus pares de la otra modalidad. Adicionalmente, se tomaron como variables moderadoras del efecto de la modalidad semipresencial el tipo de evaluación, a saber, evaluación continua o evaluación única, y las materias del curso. Estas últimas fueron clasificadas por los autores en STEM (science, technology, engineering, mathematics) y no STEM. Dentro del primer grupo, se incluyen cursos como álgebra, estadística, química, ingeniería, por mencionar algunos, y en el segundo, cursos de enfermería, negocios, psicología, ciencias sociales, idiomas, entre otros. No se encontraron diferencias significativas entre las distintas formas de evaluación. Sin embargo, sí se hallaron diferencias significativas según la materia del curso, a favor de las disciplinas STEM. Es decir, entre los estudiantes inscritos en la modalidad semipresencial, aquellos que llevaban cursos STEM obtuvieron mejores calificaciones que quienes llevaron cursos no STEM. Por lo tanto, la materia del curso es una variable moderadora de los resultados de los estudiantes de la modalidad semipresencial. Los autores explican que esta diferencia se debe a que en los cursos STEM el conocimiento se construye con teorías bien establecidas y a partir de una argumentación lineal, mientras que en los cursos no STEM, el conocimiento requiere de diversas perspectivas que dialoguen entre sí y se construye a partir del análisis, síntesis e interpretación de las experiencias humanas, las cuales no siguen una lógica lineal necesariamente. Estos autores explican que la diferencia entre materias podría estar asociada a que en los cursos semipresenciales no se fomenta lo suficiente el diálogo y la construcción conjunta de conocimiento, por lo cual las materias no STEM no se favorecen de la modalidad.

A nivel de cursos no STEM, Abasto (2009) realizó un estudio comparativo con estudiantes de una universidad de Buenos Aires de la modalidad presencial y la semipresencial inscritos en un curso de ecología, tomando como variable su rendimiento académico. La autora también comparó las características de los alumnos. Encontró que la edad promedio y el porcentaje de estudiantes que trabajan son mayores en la modalidad semipresencial. No se encontraron diferencias significativas entre las notas de los estudiantes de ambas modalidades. 
A pesar del incremento de los cursos impartidos bajo la modalidad semipresencial y la buena recepción de los mismos en espacios de educación superior alrededor del mundo, en el Perú se han realizado muy pocas investigaciones sobre el tema. Calderón (2018) llevó a cabo un estudio con 200 estudiantes que cursaban un curso de inglés en la modalidad semipresencial. Los participantes fueron estudiantes de derecho y ciencias políticas de una universidad privada de la ciudad de Lima. En el estudio, se realizaron dos encuestas: una que indagó por la percepción de la modalidad y otra que exploró lo que los estudiantes pensaban sobre el avance de su proceso de aprendizaje en el curso. Se encontró una correlación entre los ítems de ambas encuestas. Se concluye que hay, por lo menos, algún tipo de relación entre la modalidad semipresencial y el aprendizaje de los estudiantes.

Por su lado, Matzumura y Gutiérrez (2016) llevaron a cabo un estudio con 91 médicos residentes inscritos en el curso de gerencia de la salud de una universidad pública de Lima. Dicho curso cuenta con el apoyo de una plataforma virtual además de clases presenciales, por lo tanto respondería a una modalidad semipresencial es su definición más amplia. El objetivo de esta investigación fue recoger las percepciones de los estudiantes acerca del uso de las TIC en su aprendizaje en general y sobre el aula virtual en particular, para lo cual los autores construyeron un cuestionario. A nivel descriptivo, se encontró que la mayoría de los estudiantes hace uso de internet, redes sociales, correo electrónico y bibliotecas virtuales en su proceso de aprendizaje. En relación con el aula virtual, la mayoría de los participantes califican los distintos aspectos de la misma con un 4 en una escala del 1 al 5, siendo 1 muy deficiente y 5 excelente. Además, 83\% recomienda la implementación de aulas virtuales y $90 \%$ la consideró importante en su formación como médicos especialistas.

Como se puede apreciar, en estas investigaciones con población peruana hay un énfasis en la percepción de los participantes ya sea acerca de la modalidad semipresencial específicamente o de los recursos virtuales en su aprendizaje en general. Al abordar los aprendizajes de los estudiantes, las investigaciones recaen nuevamente en la percepción que tienen ellos mismos sobre su proceso, omitiendo otro tipo de medidas como las calificaciones, por ejemplo, que a pesar de que son indicadores cuestionables (Vo et al., 2017), no dejan de ser medidas más objetivas. Por otro lado, no se encuentran estudios comparativos, lo cual dificulta sacar conclusiones acerca de la efectividad de las diferentes modalidades en la población nacional. Esto último es una tarea fundamental para tomar decisiones respecto a la implementación de la misma en el contexto peruano de educación superior. Ante este panorama surge la presente investigación, que tiene como objetivo medir los resultados 
de aprendizaje objetivos, a través de las calificaciones, de dos grupos similares en cuanto a características de la población y del docente que fueron dictados en dos modalidades diferentes. Por todo ello, el objetivo general de este estudio es describir las diferencias entre los resultados obtenidos en los cursos Estadística Descriptiva y Probabilidades e Individuo y Medio Ambientes según sus modalidades presenciales y semipresenciales (blended).

\section{Metodología}

La presente investigación es de tipo descriptivo ya que tiene por objetivo describir los resultados obtenidos por los estudiantes del curso de Estadística Descriptiva y Probabilidades y el curso de Individuo y Medio Ambiente en sus modalidades presencial y semipresencial en dos semestres consecutivos (Hernández, Fernández y Baptista, 2014). Para esta investigación se consideran las calificaciones, tanto parciales como finales.

Es importante también mencionar que se controló la variable docente, es decir, en ambos cursos fue el mismo docente quien dictó en ambas modalidades. Así mismo, el docente elegido para esta investigación es también el docente coordinador del curso y tenía ya experiencia en el dictado presencial y semipresencial. Ello tuvo como finalidad evitar que la experiencia del docente pueda afectar los resultados de la investigación tanto en una como en la otra modalidad.

\subsection{Muestra}

La población con la que se trabajó fueron alumnos de la Universidad Tecnológica del Perú matriculados en los cursos de Estadística Descriptiva y de Probabilidad e Individuo y Medio Ambiente. En un ciclo regular estos son aproximadamente 8100 estudiantes, con una edad promedio de 23 años. Así mismo, se sabe que el $40,40 \%$ de los estudiantes es mujer y el resto son hombres.

El muestreo fue intencional y no probabilístico. Se eligieron esos grupos por los profesores. Ambos grupos, tanto el blended como el presencial tenían el mismo docente. Esto se hizo con la intención de eliminar al docente como variable que pueda influir en los resultados de la investigación. La muestra con la que se trabajó mantenía la misma proporción entre hombres y mujeres y una edad media similar a aquella de la población general. En la siguiente tabla se detallan las secciones y la cantidad de alumnos que estudiaba en cada una de las modalidades. Cabe mencionar que esta es la muestra final con la que se trabajó. 
Tabla 1. Detalles de la muestra

\begin{tabular}{cccc}
\hline Ciclo & Cantidad & $\begin{array}{c}\text { Estadística descriptiva } \\
\text { y de probabilidad }\end{array}$ & $\begin{array}{c}\text { Individuo y medio } \\
\text { ambiente }\end{array}$ \\
\hline \multirow{2}{*}{2017 Marzo } & Alumnos presencial & 36 & 30 \\
& Alumnos blended & 27 & 13 \\
\hline \multirow{2}{*}{2017 Agosto } & Alumnos presencial & 33 & 35 \\
& Alumnos blended & 29 & 20 \\
\hline
\end{tabular}

\subsection{Procedimientos}

Para el desarrollo de esta publicación se analizaron las bases oficiales de la universidad, correspondiente a las notas y los datos de filiación de los alumnos en los cursos seleccionados (promedio final y notas parciales).

Para el análisis de los datos, se procedió a crear una base de datos en SPSS para realizar las comparaciones respectivas. Dado que los cursos tenían sistemas de evaluación distintos, como se detallará más adelante, se crearon dos bases, una para cada curso, y se manejaron por separado.

Luego, dentro de cada curso se realizó una comparación de medias que buscaba saber si existían diferencias estadísticamente significativas en los promedios finales de los alumnos según la modalidad. En este momento se notó que una cantidad importante de alumnos no habían rendido todas las evaluaciones y ello alteraba las comparaciones al afectar significativamente los promedios. Por esta razón, el siguiente paso fue la eliminación de los alumnos que hubieran rendido menos de la mitad de las evaluaciones o que no hubieran presentado el examen final. Casos que fueron considerados dentro de la categoría «retirados». La muestra que se presenta en la sección anterior considera el número final de alumnos con los que se trabajó luego de la depuración.

Habiendo hecho la depuración de los alumnos de las bases de datos, se volvió a realizar los análisis de las notas de los alumnos. Luego, se compararon las notas de las distintas evaluaciones que se rinden a lo largo del curso.

\section{Resultados}

Antes de analizar los resultados es importante mencionar algunas características generales de los cursos. Todos los cursos en modalidad semipresencial siguen un proceso de creación a través de un diseño instruccional. Estos cursos cuentan con actividades virtuales semanales que el alumno debe realizar previamente a las clases presenciales, que también son semanales. Las actividades 
virtuales incluyen videos, lecturas y autoevaluaciones. Así mismo, ambos cursos tienen videoconferencias cuya finalidad es que el docente pueda resolver algunas dudas específicas de los alumnos. En el caso del curso de Estadística Descriptiva y Probabilidades, los alumnos también cuentan con foros de debate y materiales complementarios para el estudio individual. Así mismo, ambos cursos cuentan con foros de consulta disponibles en todo momento a través del LMS de la institución y actividades grupales; sin embargo, estas se desarrollan en los momentos presenciales. En cuanto a los cursos presenciales, estos no cuentan con materiales estandarizados y es potestad del profesor crear sus propios materiales basándose en los lineamientos del sílabo. Los sílabos de los cursos presenciales y virtuales son exactamente iguales en tanto logro, contenido y fórmula de evaluación.

Los resultados se describirán siguiendo los objetivos del estudio. El primer objetivo es comparar las notas finales de los estudiantes de las modalidades presencial y semipresencial. Para ello, primero, se buscó determinar la normalidad de las muestras. Se encontró que en ninguno de los dos cursos las notas seguían una distribución normal. Por ello se usó la prueba no paramétrica de U-de Mann-Whitney que arrojó que no había diferencias estadísticamente significativas según modalidad. En la tabla 2 se muestran las notas promedio de ambos cursos y ambos ciclos según modalidad.

Tabla 2. Comparaciones de notas entre modalidades

\begin{tabular}{lcccc}
\hline & \multicolumn{2}{c}{ Ciclo 2017-1 } & \multicolumn{2}{c}{ Ciclo 2017-2 } \\
\hline & $\begin{array}{c}\text { Promedio } \\
\text { semipresencial }\end{array}$ & $\begin{array}{c}\text { Promedio } \\
\text { presencial }\end{array}$ & $\begin{array}{c}\text { Promedio } \\
\text { semipresencial }\end{array}$ & $\begin{array}{c}\text { Promedio } \\
\text { presencial }\end{array}$ \\
\hline $\begin{array}{l}\text { Estadística Descriptiva } \\
\text { y de Probabilidad }\end{array}$ & 13.67 & 12.89 & 13.66 & 14.30 \\
\hline $\begin{array}{l}\text { Individuo y Medio } \\
\text { Ambiente }\end{array}$ & 13.00 & 13.00 & 12.75 & 12.97 \\
\hline
\end{tabular}

Luego de realizar las comparaciones entre los promedios finales de los cursos en ambas modalidades, se llevó a cabo la comparación entre cada una de las evaluaciones. Cada curso cuenta con un sistema de calificación distinto diseñado para identificar si las competencias que se espera que el alumno obtenga con este curso han sido logradas. Tal como se mencionó anteriormente, se crearon bases de datos distintas para ambos cursos y se manejaron por separado. 
Tabla 3. Sistema de evaluación Estadística Descriptiva y de Probabilidad

\begin{tabular}{lcc}
\hline \multicolumn{1}{c}{ Evaluación } & Peso & Descripción \\
\hline Prueba de entrada & $0 \%$ & Individual \\
Practica calificada 1 & $15 \%$ & Individual \\
Trabajo aplicativo & $15 \%$ & Grupal \\
Practica calificada 2 & $20 \%$ & Individual \\
Practica calificada 3 & $20 \%$ & Individual \\
Examen final & $30 \%$ & Individual \\
\hline
\end{tabular}

En cuanto a las evaluaciones del curso de Estadística, cabe mencionar que todas las prácticas calificadas tienen la misma estructura. Estas cuentan con dos preguntas teóricas sobre los conceptos correspondientes al tema de la unidad y tres ejercicios prácticos en donde se espera que los alumnos resuelvan las operaciones correspondientes e interpreten los resultados, y el examen final tiene un formato similar al de las prácticas. Mientras que el trabajo grupal es una investigación en donde se les pide a los alumnos una reflexión sobre algún contenido del curso, este trabajo también debe ser expuesto y debatido en clase.

En las comparaciones correspondientes a las distintas evaluaciones del semestre solo se encontró una diferencia significativa (sig. .0003) en la tercera práctica calificada del ciclo 2017-2. Esta evaluación mide los temas correspondientes a la unidad tres: Probabilidad condicional.

Por otra parte, a continuación, en la tabla 4 se presenta el sistema de calificación del curso Individuo y Medio Ambiente.

Tabla 4. Sistema de evaluación Individuo y Medio Ambiente

\begin{tabular}{lcc}
\hline \multicolumn{1}{c}{ Evaluación } & Peso & Descripción \\
\hline Practica calificada 1 & $20 \%$ & Individual \\
Practica calificada 2 & $20 \%$ & Individual \\
Promedio de tareas parciales & $20 \%$ & Individual \\
Trabajo final & $20 \%$ & Individual \\
Examen final & $20 \%$ & Individual \\
\hline
\end{tabular}

Las prácticas calificadas del curso Individuo y Medio Ambiente incluyen una evaluación escrita a base de preguntas para desarrollar sobre los temas de la unidad y la resolución de tareas prácticas en las semanas previas a dicha evaluación. El promedio de tareas parciales incluye pequeñas tareas que 
sirven de complemento a los temas de clase durante las semanas comprendidas entre la segunda práctica calificada y el examen final. El trabajo final es una monografía sobre una problemática medioambiental en donde se presente una posible solución. El examen final es escrito y corresponde a la resolución de preguntas en donde se espera una reflexión de parte del alumno.

En cuanto a si se encontraron diferencias significativas en las notas, se dio que estas solo estaban presentes en el trabajo final (sig. .01). Sin embargo, al igual que en Estadística, las diferencias no son estables en ambos ciclos.

Finalmente es importante también mencionar los resultados obtenidos por los docentes en relación opinión de los alumnos. El profesor de Estadística Descriptiva y Probabilidades obtuvo 18,3/20 y 18,7/20 en ambos semestres considerados en esta investigación. La profesora de Individuo y Medio Ambiente, por su parte obtuvo 18,7/20 y 17/20. Ambas notas son superiores al promedio de los profesores de la universidad que es 16,97 y 16,24 en los semestres de marzo y agosto respectivamente.

\section{Discusión}

Los cursos propósito de este estudio, tanto en su versión semipresencial y en su versión presencial, pasan por un proceso de diseño o planificación cuya finalidad es lograr que se cumplan los logros planteados. En particular, en el caso de los cursos semipresenciales se considera qué aspectos son mejores aprender en los espacios virtuales y cuáles otros en los momentos presenciales, lo que significa que cumple con las características de las investigaciones citadas en el marco conceptual.

Sobre este marco, los resultados en notas finales de los cursos semipresenciales concuerdan con la literatura revisada (Larson y Chung-Hsien, 2009; Vo et al., 2017) en tanto que no se encontraron diferencias significativas en las calificaciones de los alumnos según su modalidad.

Sin embargo, contrario a lo encontrado en Vo et al. (2017), no se encontraron diferencias significativas entre las notas de los alumnos según la materia. En este estudio se trabajó con un curso STEM, Estadística Descriptiva y de Probabilidad, y un curso no STEM, Individuo y Medio Ambiente. El hecho de no haber encontrado diferencias entre ambos puede deberse a que, si bien el curso Individuo y Medio Ambiente no es un curso STEM, según la definición de Vo et al., al tener una organización lineal podría coincidir con la principal cualidad del STEM en los estudios con cursos virtuales o blended. Del mismo modo, en un estudio realizado en Argentina con un curso similar a Individuo y Medio Ambiente tampoco se encontró diferencias significativas 
en los resultados académicos de los alumnos de la modalidad presencial y los de la modalidad semipresencial (Abasto, 2009). Esto también podría deberse a que los cursos que buscan fomentar el pensamiento crítico en los estudiantes sí se beneficia de la modalidad semipresencial. Como afirma la literatura, los cursos semipresenciales pueden favorecer el pensamiento crítico y la participación de los alumnos a través de los foros ofreciendo así una ventaja sobre las clases presenciales (Larson y Chung-Hsien, 2009). Se argumenta que la herramienta foro, utilizada en ambos cursos de esta investigación, facilita la participación de los estudiantes, sobre todo de aquellos que no suelen participar en las clases, ya sea por la premura del tiempo o por timidez.

A pesar de que no se encontraron diferencias significativas entre las notas finales de los estudiantes, sí se encontraron diferencias entre las notas de algunas de las notas parciales. Es importante resaltar que la diferencia solo se da en uno de los dos ciclos estudiados. Por lo que podría concluirse que estás diferencias podrían deberse a factores externos a este estudio, no controlados. En ambos cursos, las diferencias favorecen al grupo presencial, obteniendo notas mayores que el grupo semipresencial.

Recordamos que en el curso de Estadística Descriptiva y Probabilidades se encontró una diferencia en la práctica calificada correspondiente al tema de probabilidad condicional. Del mismo modo, en el curso Individuo y Medio Ambiente se encontró una diferencia significativa en las notas del trabajo final. Este trabajo es una monografía integradora en donde se busca que el alumno consolide la información brindada en el curso y lo contextualice en la presentación de posibles soluciones a un problema medioambiental. En la literatura no se ha encontrado mayores referencias sobre estos detalles particulares; sin embargo, podemos hacer referencias al estudio de Vo et al., (2017) donde se afirma que no existen diferencias significativas en las notas de los alumnos según los tipos de evaluaciones que pueden ser continuas o únicas, según el curso.

Por último, es importante mencionar que, si bien para este estudio se buscó controlar algunas variables, especialmente aquellas referentes a las capacidades tanto teóricas, pedagógicas y tecnológicas del docente, este estudio no es una intervención. Por el contrario, se buscó respetar el desarrollo natural de un curso ya que el objetivo no es medir los resultados en una realidad ideal.

\section{Conclusiones}

La primera conclusión a la que se puede llegar con este trabajo es que no existen diferencias significativas en cuanto a las notas finales de los alumnos de ambos cursos estudiados, Estadística Descriptiva y Probabilidades e Individuo 
y Medio Ambiente. Ello está en concordancia con lo descrito en la literatura. Estos resultados nos permiten seguir apostando por las modalidades semipresenciales, puesto que es justo lo que se busca, obtener el mismo logro. Diferentes caminos, pero la misma meta.

El no obtener diferencias significativas entre los resultados de un curso y el otro, curso STEM y no STEM en la modalidad semipresencial, debería llevarnos a seguir analizando y evaluar la forma en que el curso no STEM está diseñado. Podría deberse a que, si bien recibe la etiqueta de curso de Humanidades, los procesos que se siguen para desarrollar el aprendizaje podrían ser bastante lineales. Entendemos que ambos cursos buscan la reflexión crítica y como lo demuestra la literatura, se sabe que el pensamiento crítico es una capacidad que se ve favorecida por el aprendizaje semipresencial.

Es importante continuar las investigaciones sobre los resultados de aprendizaje según diferentes modalidades. En nuestro país, muchas universidades empiezan a ofrecer en un mismo curso opciones presenciales, semipresenciales y virtuales. ¿De qué forma podemos saber que son diferentes modalidades y metodologías que deberían llevar hacia un mismo fin? El aprendizaje medido objetivamente puede convertirse en un indicador de control de calidad.

Finalmente, es pertinente también ahondar en la investigación sobre el nivel de satisfacción de los estudiantes según la modalidad puesto que ello podría ayudar a las instituciones a tomar decisiones futuras. En este estudio se encontró que los profesores obtuvieron notas superiores al promedio; sin embargo, sugerimos seguir explorando en la percepción de los estudiantes y considerar también metodologías cualitativas que ofrezcan mayor detalle sobre la experiencia del alumno en este tipo de cursos.

\section{REFERENCIAS BIBLIOGRÁFICAS}

Abasto, P. (2009). La enseñanza de la Ecología en un nuevo ambiente de aprendizaje. Resultados de una experiencia de curso semipresencial en la carrera de ingeniería agronómica. Formación Universitaria, 2(6), 15-20. https://doi. org/10.4067/S0718-50062009000600003

Al-Qahtani, A. A. y Higgins, S. E. (2012). Effects of traditional, blended and e-learning on students' achievement in higher education. Journalof Computer Assisted Learning, 29(3), 220-234. https://doi.org/10.1111/j.1365-2729. 2012.00490.x

Azevedo, R. y Aleven, V. (2013). Metacognition and Learning Technologies: An Overview of Current Interdisciplinary Research. En R. Azevedo y V. Aleven (eds.), International Handbook of Metacognition and 
Learning Technologies (pp. 1-16). Nueva York: Springer. https://doi. org/10.1007/978-1-4419-5546-3

García-Aretio, L. (2018). Blended learning y la convergencia entre la educación presencial y a distancia. RIED. Revista Iberoamericana de Educación a Distancia, 21(1), 9-22. https://doi.org/10.5944/ried.21.1.19683

García-Aretio, L. (2020). Bosque semántico: ¿educación/enseñanza/aprendizaje a distancia, virtual, en línea, digital, eLearning...? RIED. Revista Iberoamericana de Educación a Distancia, 23(1), 9-28. https://doi. org/10.5944/ried.23.1.25495

García-Ruiz, R., Aguaded, I. y Bartolomé-Pina, A. (2018). La revolución del blended learning en la educación a distancia. Revista Iberoamericana de Educación a Distancia, 21(1), 25-32. https://doi.org/10.5944/ried.21.1.19803

Graham, C. R. (2006). Blended learning systems. Definition, current trends, and future directions. En Handbook of blended learning: Global perspectives, local designs. (pp. 3-21). San Francisco, CA: Pfeiffer.

Hernández, R., Fernández, C. y Baptista, P. (2014). Metodología de la investigación (6a. ed.). México D.F.: McGraw-Hill.

Khan, S. (2015). Blended Learning vs Traditional Classroom Settings. International Journal of Nursing, 2(1), 158-161. https://doi.org/10.15640/ijn.v2n1a17

Larson, D. y Chung-Hsien, S. (2009). Comparing Student Performance: Online versus Blended versus Face-to-Face. Journal of Asynchronous Learning Networks, 13(1), 31-42. https://doi.org/10.24059/olj.v13i1.1675

Matzumura, J. y Gutiérrez, H. (2016). Utilización de tecnología, información, comunicación y aula virtual en la enseñanza de la asignatura de Gerencia en Salud para los médicos residentes de la Facultad de Medicina de la Universidad Nacional Mayor de San Marcos, 2015. Anales de la Facultad de Medicina, 77(3), 251-256. https://doi.org/10.15381/anales.v77i3.12411

Mortis, S., Del Hierro, E., García, R. y Manig, A. (2015). La modalidad mixta: un estudio sobre los significados de los estudiantes universitarios. Innovación Educativa, 15(68), 73-97.

Pei-Chen, S., Tsai, R., Finger, G., Chen, Y. y Yeh, D. (2008). What drives a successful e-learning? An empirical investigation of the critical factors influencing learner satisfaction. Computers \& Education, 50(4), 1183-1202. https://doi.org/10.1016/j.compedu.2006.11.007

Vo, M. H., Zhu, C. y Diep, A. N. (2017). The effect of blended learning on student performance at course-level in higher education: A meta-analysis. Studies in Educational Evaluation, 53, 17-28. https://doi.org/10.1016/ j.stueduc.2017.01.002 
Ana Dasso Vassallo e Inés Evaristo Chiyong

Zhang, W. y Zhu, C. (2017). Review on Blended Learning: Identifying the Key Themes and Categories. International Journal of Information and Education Technology, 7(9), 673-678. https://doi.org/10.18178/ ijiet.2017.7.9.952 